\title{
Obesity and Kidney Transplant Candidates: How Big Is Too Big for Transplantation?
}

\author{
Krista L. Lentine ${ }^{a, b}$ Rowena Delos Santos ${ }^{c}$ David Axelrod $^{d}$ Mark A. Schnitzler ${ }^{a, b}$ \\ Daniel C. Brennan ${ }^{c}$ Janet E. Tuttle-Newhall ${ }^{b}$ \\ ${ }^{a}$ Center for Outcomes Research, Saint Louis University School of Medicine, ${ }^{b}$ Kidney and Pancreas Transplant Program, \\ Saint Louis University School of Medicine, and 'Division of Nephrology, Washington University School of Medicine, \\ St. Louis, Mo., and d Department of Surgery, Dartmouth-Hitchcock Medical Center, Hanover, N.H., USA
}

\section{Key Words}

Body mass index $\cdot$ Cardiovascular disease $\cdot$ Health care costs $\cdot$ Kidney transplantation $\cdot$ Mortality $\cdot$ Obesity

\begin{abstract}
Obesity impacts many inter-related, and sometimes conflicting, considerations for transplant practice. In this article, we describe an approach for applying available data on the importance of body composition to the kidney transplant population that separates implications for candidate selection, risk stratification among selected candidates, and interventions to optimize health of the individual. Transplant recipients with obesity defined by elevated body mass index (BMI) have been shown in many (but not all) studies to experience an array of adverse outcomes more commonly than normalweight transplant recipients, including wound infections, delayed graft function, graft failure, cardiac disease, and increased costs. However, current studies have not defined limits of body composition that preclude clinical benefit from transplantation compared with long-term dialysis in patients who have passed a transplant evaluation. Formal cost-effectiveness studies are needed to determine if payers and society should be compensating centers for clinical and financial risks of transplanting obese end-stage renal disease patients. Recent studies also demonstrate the limitations of BMI alone
\end{abstract}

as a measure of adiposity, and further research should be pursued to define practical measures of body composition that refine accuracy for outcomes prediction. Regarding individual management, observational registry studies have not found beneficial associations of pretransplant weight loss with patient or graft survival. However, association studies cannot distinguish purposeful from unintentional weight loss as a result of illness and comorbidity. Prospective evaluations of the impact of targeted risk modification efforts in this population including dietary changes, monitored exercise programs, and bariatric surgery are urgently needed.

Copyright $\odot 2012$ S. Karger AG, Basel

\section{The Obesity Epidemic and Kidney Transplantation}

As obesity has reached epidemic proportions in the general population [1], achievement and maintenance of healthy body weight have been prioritized in the United States (US) national health promotion goals [2]. The obesity epidemic and its health consequences have not spared the population with end-stage renal disease (ESRD). Obesity impacts many inter-related considerations for transplant practice including candidate selection, outcomes prediction before and after transplant, and waitlist management.

\section{KARGER \\ Fax +4161306 1234 \\ E-Mail karger@karger.ch}

www.karger.com (c) 2012 S. Karger AG, Basel

0250-8095/12/0366-0575\$38.00/0

Accessible online at:

www.karger.com/ajn
Krista L. Lentine, MD, MS

Saint Louis University Center for Outcomes Research, Salus Center 4th Floor 3545 Lafayette Avenue

St. Louis, MO 63104 (USA)

E-Maillentinek@slu.edu 
Fig. 1. The obesity epidemic: changing BMI distribution of kidney transplant recipients in the US over time. Based on review of OPTN Standard Transplant Analysis Research (STAR) files by Lentine and Xiao (file date December 2011). BMI was categorized by WHO criteria as: underweight (<18.5), normal weight (18.5-24.9), overweight (25-29.9), obese (BMI 3034.9), morbidly obese (BMI 35-39.9), and very morbidly obese (BMI $\geq 40$ ).

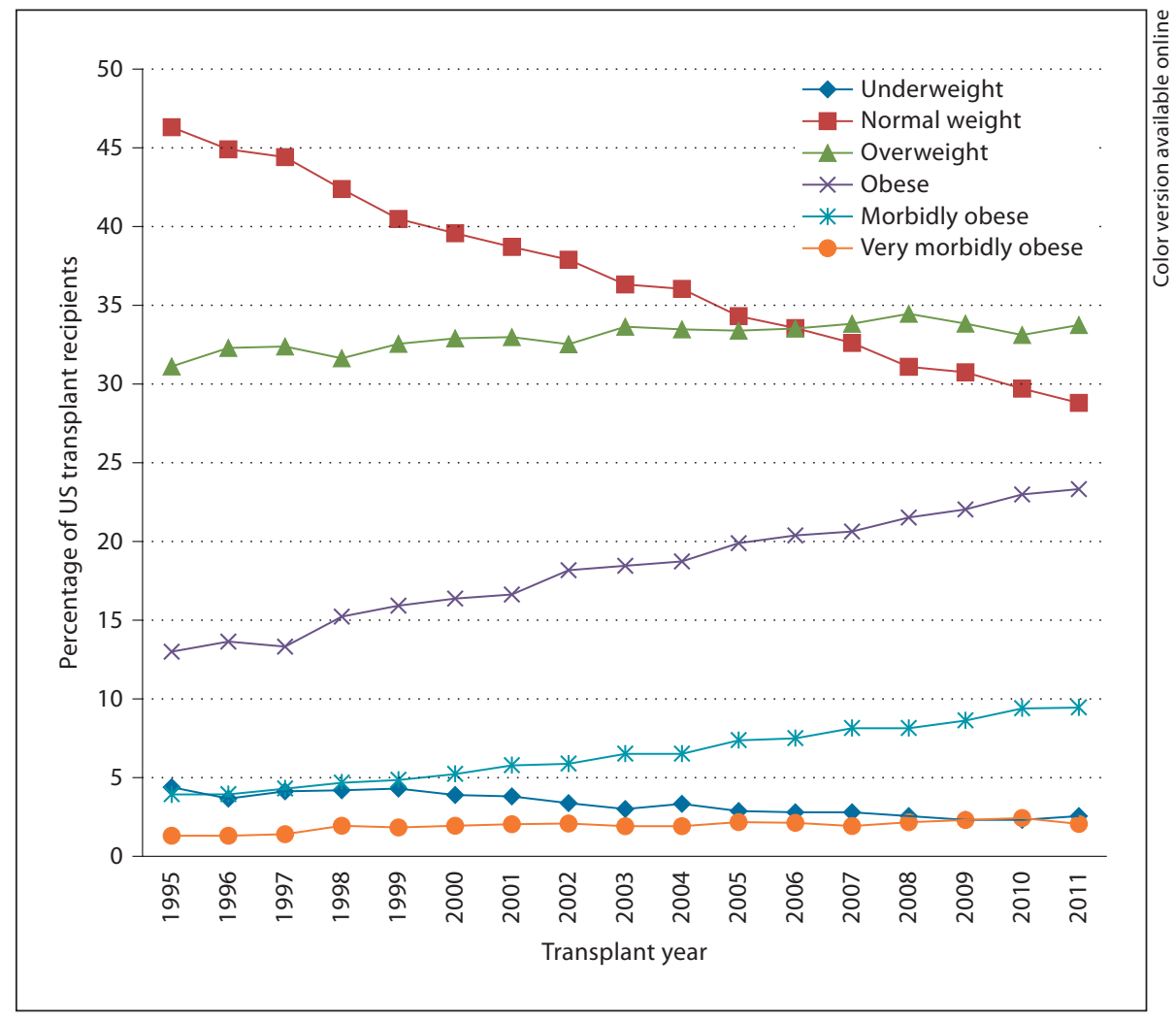

Existing practice guidelines issued by the American Society of Transplantation recommend a supervised weight loss regimen including a low-calorie diet, behavioral therapy, and a physical activity plan to achieve a goal body mass index (BMI) of less than 30 prior to kidney transplantation [3]. These guidelines also note that there are insufficient data to suggest which, if any, obese patients should be denied a transplant based on their obesity [3]. The Canadian Transplant Society has released similar guidelines, although their statement regarding the lack of data for exclusion based on obesity is placed before their recommendation for weight reduction [4]. As a result of the lack of clear evidence, acceptable BMI limits for kidney transplant candidates vary across transplant centers. A recent survey of kidney transplant programs by the American Society of Transplant Surgeons found that, among the 67 centers that responded, 66 used BMI as a selection criterion, with a range of 35-45 as the upper limit to initiate an evaluation [5]. In practice, accepted BMI levels at transplant are rising to accommodate the increasing prevalence of obesity. For instance, in the early 1990s, less than $20 \%$ of candidates were classified as having any level of obesity (BMI
230) [6]. In 2011, 23\% of US recipients were classified as obese (BMI 30-34.9), 9.4\% were morbidly obese (BMI $35-39.9)$, and $2.1 \%$ were very morbidly obese (BMI $\geq 40$; fig. 1).

A dilemma arises for transplant practitioners who desire normal weight levels in their transplant candidates but lack clear evidence to support a specific BMI threshold for exclusion from transplant candidacy. In the current article, we describe an approach for considering available data on the importance of body composition to the kidney transplant population that separates implications for candidate selection, risk stratification among selected candidates, and interventions to optimize health of the individual.

\section{Rationale for Use of BMI in Candidate Selection}

\section{BMI and Peritransplant Complications}

The rationale for BMI-based selection of transplant candidates articulated in the American Society of Transplantation guideline is that 'obese kidney transplant recipients have increased risk for CVD [cardiovascular dis- 
ease] and surgical complications' compared with normalweight transplant recipients [3]. This conclusion is supported by a study of deceased donor transplant recipients at one center in 1986-1988 [7]. Compared to recipients with $\mathrm{BMI} \leq 30$, those with obesity defined by $\mathrm{BMI}>30$ had longer operative times, prolonged hospitalizations, higher rates of reintubation, more frequent intensive care unit admissions, and a greater incidence of wound complications. More recently, an investigation of 767 privately insured transplant recipients in 2000-2007 found that mean length of stay rose from 8.4 days among normal weight to 13.3 days among very morbidly obese patients [8].

Increased risk of wound and surgical site infections (SSI) in obese patients is well established in the surgical literature [9]. Based on data for 869 kidney transplant recipients, Lynch et al. [10] reported a graded increase in the frequency of SSI with higher BMI, from $8.5 \%$ among those with BMI $20-25$ to $40 \%$ among those with BMI $>40$. Of more concern, the development of an SSI was associated with a significant increase in the risk of allograft loss at 3 years (HR 2.2, 95\% CI: 1.36-3.55).

The association of high BMI with impaired early allograft function has been established through a variety of studies. A retrospective cohort study of 51,927 US kidney transplants in 1988-1997 reported a graded association between the risk of delayed graft function (DGF) and recipient BMI [11]. Compared to those with normal weight (BMI 24-26), transplant recipients with $\mathrm{BMI}<18$ had $20 \%$ lower relative risk of DGF, while those with $\mathrm{BMI}>36$ had $51 \%$ higher relative risk. Similar results were reported in a Dutch study of transplant recipients $(n=2,067)$ in 1994-2005 [12]. DGF affected 31\% of obese compared with $21 \%$ of non-obese patients. Among a sample of privately insured transplant recipients in 2000-2007, the proportion with DGF rose from $12.0 \%$ in those with normal BMI to $36.4 \%$ among the very morbidly obese group with BMI $>40$ [8]. Finally, Molnar et al. [13] linked data from the Scientific Registry of Transplant Recipients (SRTR) with maintenance hemodialysis records from a large US dialysis organization to evaluate the relationship between BMI and DGF among 11,836 hemodialysis patients (dialyzed in 20012006) who underwent kidney transplantation by 2007. Compared with the reference BMI of 25, higher pretransplant BMI was associated with progressively higher DGF risk such that those with pretransplant BMI $>40$ had nearly 3 times the odds of DGF (adjusted OR, aOR, 2.78 , 95\% CI: 1.88-4.12) after extensive multivariate adjustment.

Obesity and Kidney Transplantation

\section{BMI and Posttransplant Allograft and Patient}

Survival

Beyond the peritransplant period, a more complex Jshaped relationship between transplant BMI and graft loss has been observed. In the study of 51,927 kidney recipients (1988-1997) with up to 10 years of follow-up, death-censored graft loss risk was modestly increased $(\sim 15 \%)$ among those with low BMI $<18$, but highest among morbidly obese patients [11]. Recipients with BMI $>36$ had 50\% higher adjusted relative risk of graft loss compared to those with BMI 24-26. Death with a functioning allograft followed a U-shaped relationship, with increased mortality at both extremes of BMI. Similar Ushaped patterns across BMI levels were found for cardiovascular and infectious disease deaths. These U patterns are similar to those observed for cardiovascular deaths, cancer deaths, and all-cause mortality in relation to BMI in the general population [14]. Another study of 2,067 transplant recipients in the Netherlands found that , compared with BMI 22-25, BMI $>25$ and BMI $>28$ were independently predictive of graft loss and patient death, respectively [12].

Importantly, weight gain after transplant is common [15]. Pretransplant obesity may exacerbate posttransplant weight gain and contribute to development of the metabolic syndrome, which has been identified in more than $50 \%$ of prevalent renal transplant recipients in some samples and associated with reductions in long-term allograft function [16]. A new study of 1,810 transplant recipients in the Netherlands with median 8 years of follow-up found that one-year posttransplant BMI and BMI change were more strongly associated with death and graft failure than pretransplant BMI [15]. Recipients with BMI $>30$ at one year after transplant had 39\% higher relative risks of both mortality and death-censored graft failure compared to those with normal BMI. In another study of 292 transplant recipients at one center, patients with an increase in BMI of more than $5 \%$ at 1 year after transplant had markedly higher risks of graft loss, with (HR 2.82, 95\% CI: 1.11-7.44) or without death censoring (HR 2.31, 95\% CI: 1.06-5.04) [17].

The association of BMI with allograft dysfunction also appears to be mediated, in part, by direct alteration of renal hemodynamics [18]. A study of 838 transplant recipients at the first transplant anniversary found associations of higher BMI with hyperfiltration. In turn, higher filtration fraction was an independent predictor of graft loss. 
Fig. 2. Five-year cumulative incidence estimates of posttransplant cardiac events at one center according to BMI rank and baseline comorbidity. Reproduced with permission from Lentine et al. [20]. AF = Atrial fibrillation; $\mathrm{CHF}=$ congestive heart failure; $\mathrm{KT}$ = kidney transplant; $\mathrm{MI}$ = myocardial infarction. a Five-year cumulative incidence of $\mathrm{CHF}, \mathrm{AF}, \mathrm{MI}$, and a composite of these events according to BMI quartile at transplant, where BMI quartiles were defined as: Q1, $\leq 22.9 ; \mathrm{Q} 2$, 23.0-26.0; Q3, 26.1-29.7; Q4, 229.8. b Five-year cumulative incidence of the composite cardiac outcome in transplant recipients with $\mathrm{BMI}$ above $(\mathrm{Q} 3-\mathrm{Q} 4)$ or below (Q1-Q2) the median, stratified by the presence or absence of reported pretransplant cardiac disease history, and then by presence or absence of diabetic ESRD.

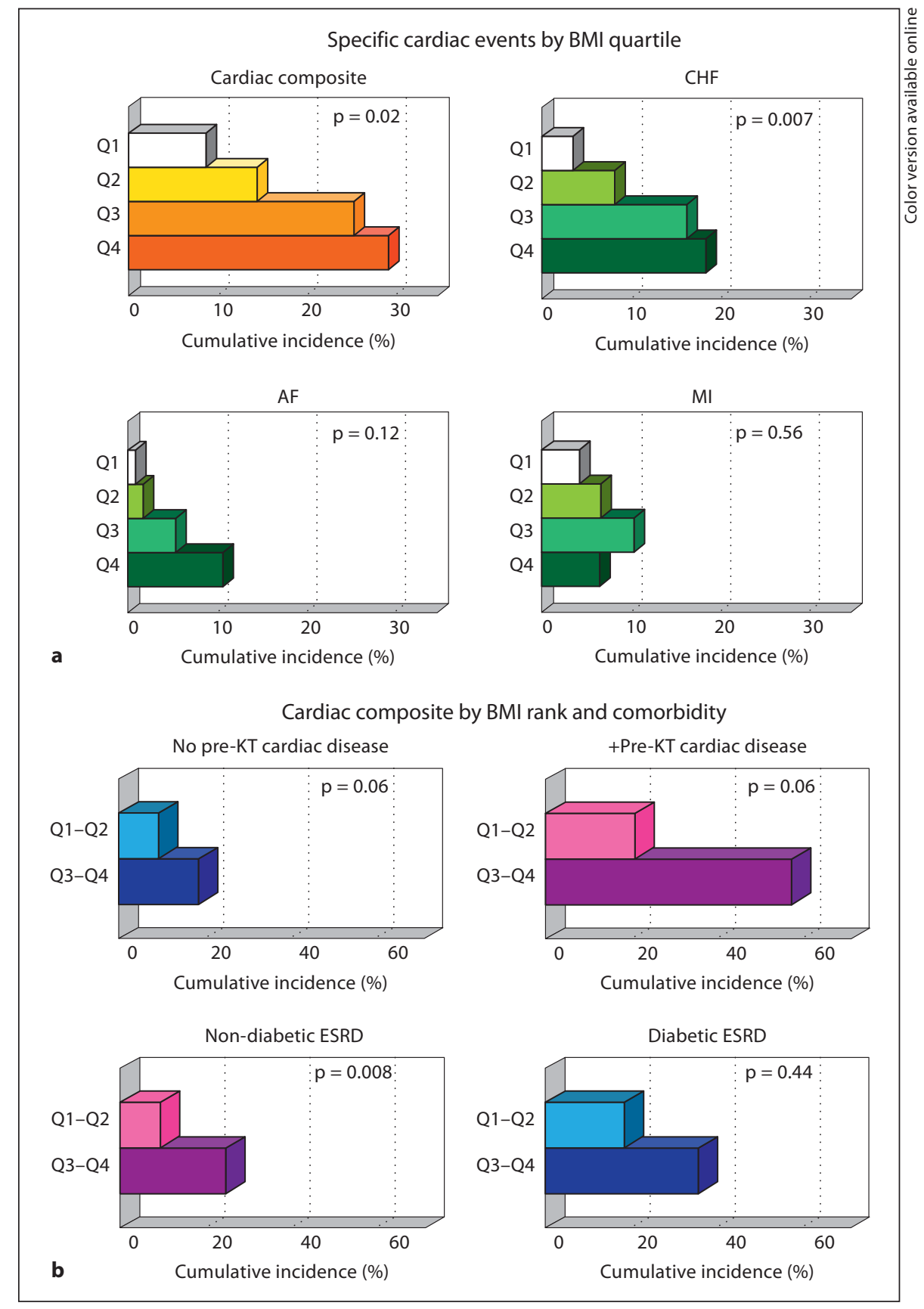

\section{BMI and Cardiovascular Risk after Transplantation}

Cardiovascular diseases comprise the most common cause of death with graft function at all time periods after kidney transplant, accounting for $30 \%$ of graft loss from death overall [19]. Lentine et al. [20] examined the relationship between BMI at transplant with posttransplant cardiac events including congestive heart failure, atrial fibrillation and myocardial infarction among 1,107 trans- plant recipients at one center. In this study, the 5-year cumulative incidence of the composite outcome of any cardiac event increased across BMI quartiles, driven primarily by the incidence of congestive heart failure and atrial fibrillation (fig. 2). In multivariate regression including adjustment for recipient, donor and transplant factors, each increase in BMI of 5 was associated with a $19 \%$ increase in the relative risk of the cardiac composite 
outcome. This study also suggested that the predictive value of BMI for posttransplant cardiac risk may be particularly important in subsets, such as those with pretransplant cardiac disease, due to accentuation of risk with high versus low BMI, and in non-diabetic patients, because elevated risk in diabetic patients at low BMI appeared to blunt BMI-related risk differential (fig. 2). Higher BMI was associated with significantly increased risk of the cardiac composite in patients with pretransplant heart disease (adjusted HR, aHR, 1.40, 95\% CI: 1.06-1.82) and in those with non-diabetic renal failure (aHR 1.33, 95\% CI: 1.08-1.64).

Overall, documentation of differing outcomes among obese compared with ideal-weight kidney transplant recipients supports a utilitarian rationale for using BMI as a criterion for transplant access. The demand for donated kidneys overwhelms the current organ supply, leading centers to make choices in their evaluation and listing practices. Although BMI is not part of allocation criteria after listing, the listing criteria of centers (which may include a maximum BMI threshold) determine who is able to 'get in line' for a transplant.

\section{Controversies Related to Use of BMI for Candidate Selection}

Controversies in use of BMI for candidate selection include considerations such as the 'reverse epidemiology' of BMI and survival on dialysis, growing recognition that $\mathrm{BMI}$ is an imperfect measure of adiposity, and evidence that obese patients selected for transplant may gain clinical benefits compared with long-term dialysis. These controversies are compounded by the lack of interventional studies targeting intentional weight loss among obese ESRD patients.

Reverse Epidemiology of BMI and Survival on Dialysis As a form of 'reverse epidemiology', associations of high BMI with lower relative mortality on dialysis have been consistently demonstrated. In a historical cohort study of 151,027 patients who initiated renal replacement therapy in the US in 1995-1997, obese patients had an unadjusted 2-year survival of $68 \%$ compared with $58 \%$ for non-obese patients [21]. Analysis of the Dialysis Outcomes and Practice Patterns Study database for European hemodialysis patients in $1996-2000$ found that $\mathrm{BMI}<20$ was consistently associated with the highest relative mortality risk [22]. Compared with a reference BMI of 2324.9, lower relative mortality was found for overweight
(BMI 25-29.9: RR 0.84, $\mathrm{p}=0.008$ ), obese (BMI 30-34.9: RR $0.73, p=0.0003$ ), and morbidly obese (BMI 35-39.9: $\mathrm{RR} 0.76, \mathrm{p}=0.02$ ) dialysis patients [22].

This apparent protective effect of higher BMI on dialysis is explained in part by underlying comorbidities and malnutrition that both reduce BMI and increase the risk of death. There is also emerging evidence that this 'reverse epidemiology' may not be present in all subgroups. For example, a new study that followed adult dialysis patients from the first dialysis treatment until 7 years, death, or kidney transplant found that while older dialysis patients had 'reverse epidemiology' for BMI, younger patients with a high BMI had 1.7 times the standardized mortality of young patients with normal BMI, mirroring typical general population patterns [23].

\section{BMI Is an Imperfect Measure of Adiposity}

Over the last decade, it has been increasingly recognized that BMI is an imperfect measure of adiposity. For example, Beddhu et al. [24] found that the protective association of high BMI with survival on hemodialysis is limited to patients with normal or high urinary creatinine excretion (considered a marker of muscle mass). In contrast, dialysis patients with high BMI, low muscle mass (based on urinary creatinine) and thus inferred high body fat had significantly increased mortality compared to those with normal BMI [24].

Recently, Streja et al. [25] examined associations of markers of pretransplant weight and muscle mass with mortality in transplant recipients using an integration of SRTR registry data with maintenance hemodialysis records for 10,090 patients who underwent transplant in 2001-2007. Considering BMI as a measure of weight and serum creatinine as a measure of muscle mass, the study found that BMI $>35$ was associated with an increased risk for graft failure in unadjusted models, but that this association was not significant after multivariate adjustment including case mix, markers of the malnutrition-inflammation complex, and transplant factors. The investigators also found that patients with high pretransplant serum creatinine (and thus inferred high muscle mass) had reduced risk of graft failure, while those with a low pretransplant serum creatinine had increased risk of graft failure. Streja et al. [25] concluded that 'pre-transplant obesity does not appear associated with poor post-transplant outcomes', but that larger muscle mass, as reflected by higher serum creatinine, is associated with graft and patient survival advantages. Pending further studies, the authors 'caution against categorical recommendation of 


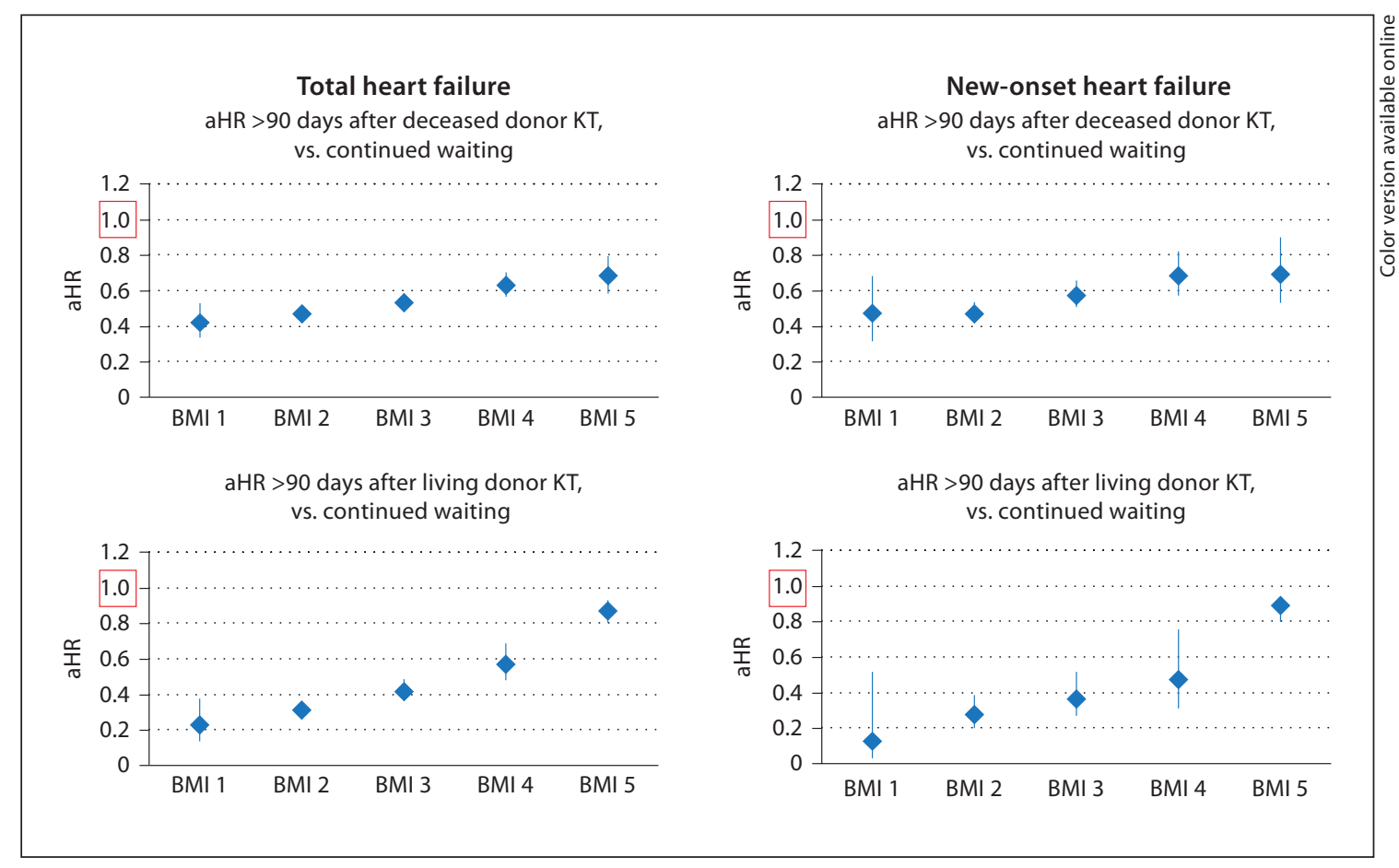

Fig. 3. Benefits of kidney transplantation on long-term heart failure risk (beyond 90 days after transplant) compared to long-term dialysis, by candidate BMI and donor type. Reproduced with permission from Lentine et al. [30]. KT = Kidney transplant. BMI was categorized into the following levels: 1 (BMI <18.5), 2 (BMI 18.524.9), 3 (BMI 25-29.9), 4 (BMI 30-34.9), 5 (BMI $\geq 35$ ). The dis- played aHRs quantify the time-dependent relative risks of heart failure (total or new onset) associated with deceased and live donor transplantation in the risk window beginning 90 days after transplant, compared with continued waiting for transplant. Reference groups for the aHR are patients in a given BMI category who remain without transplant. weight loss to apparently obese dialysis patients as a requirement for transplant wait-listing'.

While this study applies innovative data to an important topic, there are concerns about translating this conclusion into policy [26]. Because many centers consider BMI when evaluating candidacy for transplant, obese patients who receive transplants are likely selected for better than average health or undergo transplant at centers with special expertise. Importantly, by visual inspection, the association of BMI with graft loss risk remained Ushaped with all forms of adjustment, closely resembling the U-shaped association previously described by MeierKriesche et al. [11]. The confidence intervals were wide because of a small number of high-BMI patients transplanted ( $n=820)$, and the main impact of adjustment was further widening of the confidence intervals. But the study does argue that BMI alone is an imperfect measure of risk related to body composition.

Other recent studies have raised concerns about use of BMI alone for prognostication. A recent study of 993 kid- ney transplant recipients in Budapest found no clear association of BMI with long-term mortality [27]. However, when adjusted for waist circumference (a measure of abdominal fat), patients with high BMI appeared to have lower mortality compared to patients with normal, and especially low, BMI. In contrast, high waist circumference was associated with higher mortality, and this association was more pronounced after adjustment for BMI.

\section{High BMI Does Not Preclude Benefit from Kidney Transplant over Dialysis}

The use of BMI as an exclusion criterion from transplant is also controversial because of data that suggest a benefit of transplantation for high-BMI patients. Observational studies suggest that, like non-obese ESRD patients, obese patients benefit from kidney transplant in terms of lower long-term mortality and cardiovascular risk compared with continuing on the waiting list. In a study of USRDS data for 7,521 obese patients on the transplant waiting list, receipt of a transplant, when modeled 
as a time-varying covariate, was associated with a $61 \%$ reduction in the relative risk of death (HR $0.39,95 \% \mathrm{CI}$ : 0.33-0.47) compared with remaining on the waiting list [28]. The effect size was similar in the group with BMI $>40$ (HR 0.47, 95\% CI: $0.17-1.25, \mathrm{p}=0.13$ ), although the effect was not statistically significant.

Using Medicare billing claims as a marker of congestive heart failure diagnoses, Lentine et al. [29] found that deceased donor transplantation reduced the relative risk of heart failure diagnoses in the long-term, with a $54 \%$ (aHR 0.46, 95\% CI: 0.43-0.96) relative reduction in patients with normal BMI and somewhat smaller, but still significant, 32\% (aHR 0.68, 95\% CI: 0.58-0.79) reduction in morbidly obese patients (fig. 3). There was a larger differential of relative benefits across BMI strata with live donor transplantation, but significant benefit was present in all recipient BMI groups.

At least for the near future, BMI will likely continue to be used as part of kidney transplant evaluation and selection processes. The components of BMI (height and weight) are easily measured, recorded, and followed over time, and BMI is the most widely used anthropometric measure of overall body size. Further, use of BMI is recommended as part of the assessment of nutritional status for ESRD patients by current clinical practice guidelines, such as Kidney Dialysis Outcome Quality Improvement [30].

\section{Implications of Transplanting Obese Patients for the Transplant Center}

Posttransplant Outcomes Grading and the Regulation of Transplantation

Transplant in the US is an increasingly regulated field with a high level of public reporting. Centers are graded for recipient and graft survival using risk-adjusted equations developed by the SRTR for the prediction of expected one-year and 3-year posttransplant patient and graft survival [31]. Consequences of a citation for recipient death and/or graft loss rates that 'exceed expected' from the UNOS Membership and Professional Standards Committee, the Centers for Medicare and Medicaid Services or private insurance networks are serious. In addition to regulatory penalties, citations may have significant financial impacts including costly Systems Improvement Agreements with Medicare, exclusions from private payer Center of Excellence designations, loss of Medicare participation, or program closure [32]. While the SRTR equations include adjustment parameters for BMI $>25$ and $\mathrm{BMI}>30$ based on national registry data, $\mathrm{BMI}$ is an imperfect metric of body composition as discussed above, limiting the benefit of this parameter for risk adjustment. Without adjustment that adequately captures the risk associated with transplantation of obese recipients, there will continue to be significant disincentives to offering transplant to these candidates [33]. This is especially true given that center performance metrics are focused exclusively on posttransplant outcomes, and there is currently no performance metric for comprehensive survival as from start of ESRD.

\section{Cost Implications of Obesity among Transplant Recipients}

Transplant centers must manage costs of care within relatively fixed reimbursement limits. Obese recipients are likely to incur higher costs per case, due to longer operative times and increased rates of expensive complications including SSI and DGF, as summarized above. Without compensation for higher resource consumption, there are financial disincentives for centers to offer kidney transplantation to obese patients, even if payers and society benefit clinically and economically from better long-term outcomes and a lower lifetime cost of care among successful transplant recipients. A preliminary analysis of total expenditures (in 2004 USD) among Medicare-insured kidney transplant recipients (19952004) according to BMI found that morbidly obese recipients had USD 23,924 higher cumulative adjusted one-year costs, and USD 39,085 higher 3-year costs compared with normal-weight recipients [34]. By comparison, total annual Medicare expenditures in 2004 averaged USD 70,000 and USD 50,000 per person for patients on hemodialysis and peritoneal dialysis, respectively [35].

\section{Management of Obesity in Transplant Candidates and Recipients}

\section{Controversies regarding the Impact of Pretransplant} Weight Loss

The evidence base directing the management of obese transplant candidates is challenged by the lack of prospective interventional trials. A widely cited observational study of USRDS data for 124,713 kidney transplant recipients in 1990-2003 by Schold et al. [36] found that, among patients obese at listing, weight changes from listing to transplant (considered in levels from $>12 \%$ loss to $>12 \%$ gain) were not associated with graft 
loss or death after transplant. Recently, Molnar et al. [37] examined their linkage of maintenance hemodialysis records and SRTR registry data (2001-2007) to model associations of quarterly averaged weight change with mortality while awaiting transplant. Of note, patients who were transplanted after listing - half the potential sample - were excluded from this analysis. Among the included sample, the authors found that compared to hemodialysis patients with a relatively stable dry weight, the groups with 3-5 kg pretransplant weight loss had 31\% higher adjusted death risk, and those with more than $5 \mathrm{~kg}$ weight loss had $51 \%$ increased risk. In contrast, dialysis patients who gained weight before transplant had reduced mortality.

Importantly, these studies cannot distinguish intentional from unintentional weight loss as a result of illness and comorbidity. In the case of the study that excluded participants based on receipt of a transplant [37], there is inherent potential for selection bias as those who were not transplanted may be sicker. Perhaps of greater importance, the exclusion was associated with the outcome, as excluded transplant recipients are guaranteed to have survived until transplant. By simulation analysis, Segev et al. [38] illustrated that any increase in the likelihood of kidney transplant with weight loss would, in the setting of a true equal mortality, cause the appearance of higher death with weight loss as an artifact. In other words, such exclusion reduces apparent survival for the group favored for transplantation. So at this time, evidence assessing the impact of intentional pretransplant weight loss efforts among obese transplant candidates, including dietary changes and monitored exercise programs, remains an urgent need.

\section{Bariatric Surgery in Transplant Candidates and Recipients}

Meta-analyses support superior efficacy of bariatric surgery compared to non-surgical therapy in achieving sustained weight loss in morbidly obese patients in the general population $[39,40]$. A National Institutes of Health Consensus Development Conference approved clinical indications for bariatric surgery as BMI $\geq 40$, or BMI $\geq 35$ with obesity-related comorbidities such as sleep apnea, cardiomyopathy or severe diabetes [41]. Use of bariatric surgery has been relatively uncommon among kidney transplant candidates and recipients despite otherwise qualifying indications. In 1996, Marterre et al. [42] first described open gastric bypass among 3 morbidly obese kidney transplant recipients. Published experience with bariatric surgery among chronic kidney dis- ease (CKD) patients by this Ohio group includes 9 pretransplant and 10 posttransplant cases, all accomplished without perioperative mortality or graft loss [43, 44]. Mean estimated body weight loss was $69-79 \%$ by 3 years and sustained at $70 \%$ at up to 5 years. A recent examination of Medicare billing claims within USRDS registry data (1991-2004) identified 188 bariatric surgery cases among renal allograft candidates and recipients, of which 72 were performed before listing, 29 while on the waitlist, and 87 after transplant [45]. Thirty-day mortality after bariatric surgery performed while on the waitlist and after transplant was $3.5 \%$, and one transplant recipient lost the graft within 30 days after bariatric surgery. BMI data were available for a subset and suggested median excess body weight loss of 31-61\%. Comparison with published clinical trials of bariatric surgery in populations without kidney disease $[46,47]$ suggests comparable weight loss but higher postbariatric surgery mortality in the USRDS sample.

A recent analysis of the relationship of preoperative renal function with complications after bariatric surgery among 27,736 patients who underwent bariatric surgery in 2006-2008 included 34 patients who received longterm dialysis prior to surgery and a total of 185 with CKD stages 4 or 5 [48]. Complications were defined by chart review and included wound, respiratory, urinary tract, central nervous system, cardiac, and mortality. Multivariate logistic regression with adjustment for diabetes and hypertension revealed a graded increase in the likelihood of complications with advancing severity of renal dysfunction, such that those with CKD stage 5 had more than twice the odds of complications (aOR 2.07, 95\% CI: $1.04-4.15)$ as those with eGFR $>90 \mathrm{ml} / \mathrm{min} / 1.73 \mathrm{~m}^{2}$.

Notably, the procedures identified in the USRDS sample were open, such as Roux-en-Y bypass. In more recent years, laparoscopic techniques have been promoted as potentially less morbid approaches. Published case reports and case series describing outcomes and complications of bariatric surgery in patients with CKD and kidney transplants [summarized in 45] include 6 cases of laparoscopic adjustable gastric banding before or after kidney transplant without perioperative deaths [49-51]. However, some authors raised concerns about technical complications (band slippage, band erosion, obstruction, port malfunction) and high surgical revision rates after laparoscopic adjustable gastric banding [49-51], and the need to implant a foreign body may predispose to infection in immunosuppressed patients. Laparoscopic sleeve gastrectomy generally requires shorter operative times, and was originally recommended for exceptionally high- 
Table 1. Clarifying considerations in the use of BMI for selection and management of kidney transplant candidates and recipients

Use of BMI for candidate selection

Current state of knowledge

- Markers of obesity (including BMI, waist circumference) appear associated with worse outcomes (SSI, DGF, graft failure, cardiac disease prolonged length of stay, high costs) compared with markers of ideal body size in many studies [7, 8, $10-13,20]$

- However, obesity may not preclude benefit from kidney transplant over dialysis

Observational studies suggest that, like non-obese ESRD patients, obese patients benefit from kidney transplant

in terms of lower long-term mortality and cardiovascular risk compared with continued waiting on dialysis [28, 29]

Key controversies

- How does the transplant community balance optimizing organ utility and regulatory pressures to sustain high levels of posttransplant graft and patient survival with patient-centered consideration of survival compared with experience on dialysis?

Recommended foci for future study

- Defining whether there are limits of body composition that preclude clinical benefit from kidney transplant compared to continued dialysis

- Formal cost-effectiveness studies, including appropriate quality of life adjustments that capture impact of complications, to determine if payers and society should be compensating centers for clinical and financial risks of transplanting obese ESRD patients

Use of BMI for risk stratification among selected candidates

Current state of knowledge

- As above, compared to recipients with normal BMI, kidney transplant recipients with BMI $>30$ appear in many studies to face increased risk of adverse outcomes $[7,8,10-13,20]$

Key controversies

- BMI is a surrogate measure, not a perfect reflection of adiposity

- Outcomes prediction in dialysis and transplant patients may improve when BMI is combined with other measures, such as markers of muscle or waist circumference [24, 25, 27]

Recommended foci for future study

- Defining practical measures of body composition that refine accuracy for outcomes prediction, including attention to subgroups

Interventions to optimize health of the individual

Current state of knowledge

- Observational registry studies have not shown beneficial outcomes among dialysis patients who lost weight before transplantation $[36,37]$

Key controversies

- Association studies cannot distinguish intentional from unintentional weight loss as a result of illness and comorbidity, and offer little guidance on potential benefits of purposeful weight reduction

Recommended foci for future study

- Prospective evaluations of the impact of intentional weight loss efforts among obese ESRD patients including dietary changes, monitored exercise programs, and bariatric surgery

risk patients such as super obese $(\mathrm{BMI}>45)$ and patients with hepatic cirrhosis $[52,53]$. Laparoscopic sleeve gastrectomy may be particularly beneficial in transplant recipients as it is purely restrictive and does not incorporate a malabsorptive component, minimizing interference with medication absorption, although its irreversibility may potentially harm those who lose too much weight. Medicare has recently determined that regional contractors may allow coverage of the laparoscopic sleeve gastrectomy for the treatment of comorbid conditions related to obesity in Medicare beneficiaries [54], eliminating an important barrier for ESRD patients. Further study is warranted to determine if benefits of bariatric surgery outweigh risks in the ESRD population, including potential benefits in facilitating access to transplantation.

\section{Novel Surgical Approaches to Transplantation in}

\section{Obese Recipients}

In lieu of requiring weight loss to ideal targets, novel surgical approaches have been applied in attempts to reduce complications of the transplant operation itself among obese recipients. In 2009, the first reported minimally invasive robotic kidney transplant was reported in 
a morbidly obese patient [55]. In this case, a deceased donor kidney was successfully transplanted into a woman with a BMI of 41 after $11 \mathrm{~h}$ of cold ischemia time and 50 min of warm ischemia time. The allograft had immediate function, and there were no perioperative surgical complications. This group has since expanded their experience, and recently reported a series of 27 recipients of robotic kidney transplants with at least 6 months of followup [56]. DGF, wound complications, and sepsis affected single cases. Minimally invasive surgical approaches show promise for reducing perioperative complications, but require highly trained surgeons, dramatically increase the cost of the procedure, and are not broadly available. Further study is needed to quantify both the perioperative and long-term clinical and economic outcomes of minimally invasive transplant surgery in obese ESRD patients.

\section{Conclusions}

Applying available data on the importance of body composition to the kidney transplantation population may be facilitated by separating implications for candidate selection, risk stratification among selected candidates, and interventions to optimize health of the individual (table 1). With respect to the question of appropriate BMI thresholds for candidate listing, markers of increased adiposity (including BMI and waist circumference) do appear to be associated with worse posttransplant outcomes (e.g. DGF, graft failure, cardiac disease, high costs) compared with ideal body composition in most studies. However, current data have not identified limits of body composition that preclude clinical benefit from kidney transplant compared to continued waiting on dialysis. Thus, even if BMI is considered a marker of inferior posttransplant outcomes (compared with normal BMI), exclusion from transplant on the basis of BMI alone may not be appropriate from the viewpoint of the individual candidate. In contrast, the data on the cost and outcome implications of high BMI recipients for transplant centers are clear and demonstrate significant economic and regulatory risk. In determining transplant candidacy, there is a balance between optimizing the utility of the organ/posttransplant outcomes versus a patient-centered approach that considers outcomes of transplant compared with experience on dialysis. Thus, the answer to 'How big is too big for transplant?' is that it appears to depend on the experience and risk tolerance of the individual transplant center at this time. Formal cost- effectiveness studies are needed, including appropriate quality of life adjustments that capture the impact of complications, to determine if payers and society should be compensating centers for incurring these clinical and financial risks.

Regarding use of BMI for prognosis, BMI frequently shows 'reverse' associations with dialysis survival. But, as stated above, compared to recipients with normal BMI, kidney transplant recipients with elevated BMI appear in many (but not all) studies to face increased risk of adverse outcomes. BMI is a surrogate measure of adiposity, and prediction may improve when combined with other measures, such as waist circumference and measures of muscle mass. More research should be pursued to help define practical measures of body composition that refine accuracy for outcomes prediction, including attention to subgroups.

Finally, there are insufficient data to assess the impact of pretransplant interventions to optimize health through planned weight reduction among potential candidates. Listing thresholds aside, evidence does not suggest that obesity should be disregarded. Lifestyle alterations that seem reasonable to improve health outcomes (including utility of the organ) should be encouraged. Just as we require patients with alcoholic liver disease to stop drinking prior to transplant, it may be reasonable to ask kidney transplant candidates to lose excess body fat and attempt to increase lean muscle mass by becoming more physically active and modifying their diet. To provide urgently needed evidence, prospective evaluations of the impact of intentional weight loss efforts among obese ESRD patients including dietary changes, monitored exercise programs, and bariatric surgery must be pursued. Pending more evidence, achieving and maintaining healthy body composition on the basis of guidelines for nutrition in renal failure are important priorities for kidney transplant candidates and recipients.

\footnotetext{
References

1 Centers for Disease Control and Prevention. Adult Obesity Facts. http://www.cdc.gov/ obesity/data/adult.html; accessed September $25,2012$.

2 Nutrition and Weight Status. http://www. healthypeople.gov/2020/topicsobjectives2020/overview.aspx?topicid=29 (accessed September 25, 2012).

-3 Kasiske BL, Cangro CB, Hariharan S, Hricik DE, Kerman RH, Roth D, et al: The evaluation of renal transplantation candidates: clinical practice guidelines. Am J Transplant 2001;1(suppl 2):3-95.
} 
-4 Knoll G, Cockfield S, Blydt-Hansen T, Baran D, Kiberd B, Landsberg D, et al: Canadian Society of Transplantation: consensus guidelines on eligibility for kidney transplantation. CMAJ 2005;173:S1-S25.

5 Pondrom S: The AJT report: news and issues that affect organ and tissue transplantation. Am J Transplant 2012;12:1663-1664.

-6 Pirsch JD, Armbrust MJ, Knechtle SJ, D'Alessandro AM, Sollinger HW, Heisey DM, et al: Obesity as a risk factor following renal transplantation. Transplantation 1995; 59:631-633.

7 Holley JL, Shapiro R, Lopatin WB, Tzakis AG, Hakala TR, Starzl TE: Obesity as a risk factor following cadaveric renal transplantation. Transplantation 1990;49:387-389.

8 Ercole PM, Buchanan PM, Lentine KL, Burroughs TE, Schnitzler MA, Modanlou KA: Costs and outcomes of privately-insured kidney transplant recipients by body mass index. J Nephrol Therapeutic 2012;S4:003.

$\checkmark 9$ DeMaria EJ, Carmody BJ: Perioperative management of special populations: obesity. Surg Clin North Am 2005;85:1283-1289, xii.

10 Lynch RJ, Ranney DN, Shijie C, Lee DS, Samala N, Englesbe MJ: Obesity, surgical site infection, and outcome following renal transplantation. Ann Surg 2009;250:10141020.

-11 Meier-Kriesche HU, Arndorfer JA, Kaplan B: The impact of body mass index on renal transplant outcomes: a significant independent risk factor for graft failure and patient death. Transplantation 2002;73:70-74.

$\checkmark 12$ Aalten J, Christiaans MH, de Fijter H, Hene $\mathrm{R}$, van der Heijde JH, Roodnat J, et al: The influence of obesity on short- and long-term graft and patient survival after renal transplantation. Transplant Int 2006;19:901-907.

-13 Molnar MZ, Kovesdy CP, Mucsi I, Bunnapradist S, Streja E, Krishnan M, et al: Higher recipient body mass index is associated with post-transplant delayed kidney graft function. Kidney Int 2011;80:218224.

14 Calle EE, Thun MJ, Petrelli JM, Rodriguez C, Heath CW Jr: Body-mass index and mortality in a prospective cohort of U.S. adults. N Engl J Med 1999;341:1097-1105.

-15 Hoogeveen EK, Aalten J, Rothman KJ, Roodnat JI, Mallat MJ, Borm G, et al: Effect of obesity on the outcome of kidney transplantation: a 20 -year follow-up. Transplantation 2011;91:869-874.

16 de Vries AP, Bakker SJ, van Son WJ, van der Heide JJ, Ploeg RJ, The HT, et al: Metabolic syndrome is associated with impaired longterm renal allograft function; not all component criteria contribute equally. Am J Transplant 2004;4:1675-1683.

$\checkmark 17$ Ducloux D, Kazory A, Simula-Faivre D, Chalopin JM: One-year post-transplant weight gain is a risk factor for graft loss. Am J Transplant 2005;5:2922-2928.
8 Bosma RJ, Kwakernaak AJ, van der Heide JJ, de Jong PE, Navis GJ: Body mass index and glomerular hyperfiltration in renal transplant recipients: cross-sectional analysis and long-term impact. Am J Transplant 2007;7: 645-652.

19 US Renal Data System (USRDS): USRDS 2008 Annual Data Report. Atlas of ESRD, Transplantation, figure 7.31. Bethesda, National Institutes of Health, National Institute of Diabetes and Digestive and Kidney Diseases. http://www.usrds.org/2008/view/ esrd_07.asp (accessed September 25, 2012).

20 Lentine KL, Rocca-Rey LA, Bacchi G, Wasi N, Schmitz L, Salvalaggio PR, et al: Obesity and cardiac risk after kidney transplantation: experience at one center and comprehensive literature review. Transplantation 2008;86:303-312.

21 Glanton CW, Hypolite IO, Hshieh PB, Agodoa LY, Yuan CM, Abbott KC: Factors associated with improved short term survival in obese end stage renal disease patients. Ann Epidemiol 2003;13:136-143.

22 Leavey SF, McCullough K, Hecking E, Goodkin D, Port FK, Young EW: Body mass index and mortality in 'healthier' as compared with 'sicker' haemodialysis patients: results from the Dialysis Outcomes and Practice Patterns Study (DOPPS). Nephrol Dial Transplant 2001;16:2386-2394.

23 Hoogeveen EK, Halbesma N, Rothman KJ, Stijnen T, van Dijk S, Dekker FW, et al: Obesity and mortality risk among younger dialysis patients. Clin J Am Soc Nephrol 2012;7: 280-288.

24 Beddhu S, Pappas LM, Ramkumar N, Samore M: Effects of body size and body composition on survival in hemodialysis patients. J Am Soc Nephrol 2003;14:2366-2372.

25 Streja E, Molnar MZ, Kovesdy CP, Bunnapradist S, Jing J, Nissenson AR, et al: Associations of pre-transplant weight and muscle mass with mortality in renal transplant recipients. Clin J Am Soc Nephrol 2011;6: 1463-1473.

26 Lentine KL, Axelrod D, Abbott KC: Interpreting body composition in kidney transplantation: weighing candidate selection, prognostication, and interventional strategies to optimize health. Clin J Am Soc Nephrol 2011;6:1238-1240.

27 Kovesdy CP, Czira ME, Rudas A, Ujszaszi A, Rosivall L, Novak M, et al: Body mass index, waist circumference and mortality in kidney transplant recipients. Am J Transplant 2010; 10:2644-2651.

28 Glanton CW, Kao TC, Cruess D, Agodoa LY, Abbott KC: Impact of renal transplantation on survival in end-stage renal disease patients with elevated body mass index. Kidney Int 2003;63:647-653.

29 Lentine KL, Xiao H, Brennan DC, Schnitzler MA, Villines TC, Abbott KC, et al: The impact of kidney transplantation on heart failure risk varies with candidate body mass index. Am Heart J 2009;158:972-982.
30 Kidney Dialysis Outcomes Quality Initiative. Clinical Practice Guidelines for Nutrition in Chronic Renal Failure. 2000. http:// www.kidney.org/professionals/kdoqi/ guidelines_updates/nut_a10.html (accessed September 25, 2012).

31 Scientific Registry of Transplant Recipients. Risk Adjustment Models. http://www.srtr. org/csr/current/modtabs.aspx (accessed September 25, 2012).

32 Sullivan B: Beyond CMS certification: mitigating factors application and systems improvement agreements. 20th Annual UNOS Transplant Management Forum. http:// www.regonline.com/builder/site/tab3. aspx?EventID=1030813 (accessed September 3, 2012).

33 Schold JD: Unintended consequences of PSRs. Consensus Conference on Transplant Program Quality and Surveillance. http:// www.srtr.org/cctpqs/Default.asp (accessed September 25, 2012).

-34 Modanlou KA, Ercole PM, Axelrod D, Salvalaggio PR, Graff RJ, Schnitzler MA, et al: The effect of recipient body mass index on the outcome and cost in kidney transplantation. Am J Transplant 2009;9(suppl 2):207.

35 US Renal Data System (USRDS): 2012 Annual Data Report. Atlas of ESRD, Costs of ESRD, figure 11.7. Bethesda, National Institutes of Health, National Institute of Diabetes and Digestive and Kidney Diseases. http://www.usrds.org/2012/pdf/v2_ch11_ 12.pdf (accessed October 22, 2012).

- 36 Schold JD, Srinivas TR, Guerra G, Reed AI, Johnson RJ, Weiner ID, et al: A 'weight-listing' paradox for candidates of renal transplantation? Am J Transplant 2007;7:550559.

37 Molnar MZ, Streja E, Kovesdy CP, Bunnapradist S, Sampaio MS, Jing J, et al: Associations of body mass index and weight loss with mortality in transplant-waitlisted maintenance hemodialysis patients. Am J Transplant 2011;11:725-736.

- 38 Segev DL, Massie AB, Schold JD, Kaplan B: If you're not fit, you mustn't quit: observational studies and weighing the evidence. Am J Transplant 2011;11:652-653.

- 39 Buchwald H, Avidor Y, Braunwald E, Jensen MD, Pories W, Fahrbach K, et al: Bariatric surgery: a systematic review and meta-analysis. JAMA 2004;292:1724-1737.

40 Maggard MA, Shugarman LR, Suttorp M, Maglione M, Sugerman HJ, Livingston EH, et al: Meta-analysis: surgical treatment of obesity. Ann Intern Med 2005;142:547-559.

41 Gastrointestinal surgery for severe obesity. National Institutes of Health Consensus Development Conference Statement. Am J Clin Nutr 1992;55(suppl 2):615S-619S

42 Marterre WF, Hariharan S, First MR, Alexander JW: Gastric bypass in morbidly obese kidney transplant recipients. Clin Transplant 1996;10:414-419. 
-43 Alexander JW, Goodman HR, Gersin K, Cardi M, Austin J, Goel S, et al: Gastric bypass in morbidly obese patients with chronic renal failure and kidney transplant. Transplantation 2004;78:469-474.

44 Alexander JW, Goodman H: Gastric bypass in chronic renal failure and renal transplant. Nutr Clin Pract 2007;22:16-21.

-45 Modanlou KA, Muthyala U, Xiao H, Schnitzler MA, Salvalaggio PR, Brennan DC, et al: Bariatric surgery among kidney transplant candidates and recipients: analysis of the United States renal data system and literature review. Transplantation 2009;87: 1167-1173.

-46 Nguyen NT, Goldman C, Rosenquist CJ, Arango A, Cole CJ, Lee SJ, et al: Laparoscopic versus open gastric bypass: a randomized study of outcomes, quality of life, and costs. Ann Surg 2001;234:279-289, discussion 289-291.

-47 Inabnet WB, Quinn T, Gagner M, Urban M, Pomp A: Laparoscopic Roux-en-Y gastric bypass in patients with $\mathrm{BMI}<50$ : a prospective randomized trial comparing short and long limb lengths. Obes Surg 2005;15:51-57.
48 Turgeon NA, Perez S, Mondestin M, Davis SS, Lin E, Tata S, et al: The impact of renal function on outcomes of bariatric surgery. J Am Soc Nephrol 2012;23:885-894.

- 49 Weiss H, Nehoda H, Labeck B, Oberwalder M, Konigsrainer A, Margreiter R: Organ transplantation and obesity: evaluation, risks and benefits of therapeutic strategies. Obes Surg 2000;10:465-469.

50 Newcombe V, Blanch A, Slater GH, Szold A, Fielding GA: Laparoscopic adjustable gastric banding prior to renal transplantation. Obes Surg 2005; 15:567-570.

51 Buch KE, El-Sabrout R, Butt KM: Complications of laparoscopic gastric banding in renal transplant recipients: a case study. Transplant Proc 2006;38:3109-3111.

52 Gagner M, Gumbs AA, Milone L, Yung E, Goldenberg L, Pomp A: Laparoscopic sleeve gastrectomy for the super-super-obese (body mass index $>60 \mathrm{~kg} / \mathrm{m}^{2}$ ). Surg Today 2008;38: 399-403.

53 Takata MC, Campos GM, Ciovica R, Rabl C, Rogers SJ, Cello JP, et al: Laparoscopic bariatric surgery improves candidacy in morbidly obese patients awaiting transplantation. Surg Obes Relat Dis 2008;4:159-164, discussion 164-155.
54 Center of Medicare and Medicaid Studies: Decision Memo for Bariatric Surgery for the Treatment of Morbid Obesity (CAG00250R2). http://www.cms.gov/medicarecoverage-database/details/nca-decisionmemo aspx $?$ NCAId $=258 \&$ NcaName $=$ Baria tric+Surgery+for+the+Treatment+of+Morbi $\mathrm{d}+$ Obesity\&CoverageSelection $=$ National $\&$ KeyWord=obesity\&KeyWordLookUp=Title $\&$ KeyWordSearchType $=$ And $\&$ where $=$ index \&nca_id $=219 \&$ basket $=$ nca* $3 a \$ 00397 N^{*} 3 a \$$ $219 * 3 a \$$ Surgery+for+Diabetes*3a\$Open*3a $\$ N_{e w}^{*} 3 a \$ 5 \& b c=$ gAAAABAAIAAA\& (accessed September 25, 2012).

55 Giulianotti P, Gorodner V, Sbrana F, Tzvetanov I, Jeon H, Bianco F, et al: Robotic transabdominal kidney transplantation in a morbidly obese patient. Am J Transplant 2010;10: 1478-1482.

56 Tzvetanov I, Oberholzer J, Spaggiari M, Jeon H, Garcia-Roca R, Campara M, et al: Robotic kidney transplantation in obese recipients: preliminary experience. Am J Transplant 2012;12(suppl 3):72-73. 